\title{
PRECERAMIC POLYMERS FOR USE AS FIBER COATINGS
}

P. J. Heimann, Cleveland State University, Cleveland, OH; F. I. Hurwitz, D. Wheeler and J. Eldridge, NASA Lewis Research Center, Cleveland, OH; R. Baranwal, University of Michigan, Ann Arbor, MI; R. Dickerson, Case Western Reserve University, Cleveland, $\mathrm{OH}$.

\begin{abstract}
Polymeric precursors to $\mathrm{Si}-\mathrm{C}-\mathrm{O}, \mathrm{Si}-\mathrm{B}-\mathrm{N}$ and $\mathrm{Si}-\mathrm{C}$ were evaluated for use as ceramic interfaces in ceramic matrix composites. Use of the preceramic polymers allows for easy dip coating of fibers from dilute solutions of a polymer, which are then pyrolyzed to obtain the ceramic. SCS-0 fibers (Textron Specialty Materials, Lowell, MA.) were coated with polymers from three systems: polysilsesquioxanes, polyborosilazanes and polycarbosilanes. The polysilsesquioxane systems were shown to produce either silicon oxycarbide or silicon oxynitride, depending on the pyrolysis conditions, and demonstrated some promise in an RBSN matrix model system. Polyborosilazanes were shown, in studies of bulk polymers, to give rise to oxidation resistant Si-B-N ceramics which remain amorphous to temperatures of $1600^{\circ} \mathrm{C}$, and should therefore provide a low modulus interface. Polycarbosilanes produce amorphous carbon-rich $\mathrm{Si}-\mathrm{C}$ materials which have demonstrated oxidation resistance.

\section{INTRODUCTION}

Using polymeric precursors for interface coatings offers a process with potential for reduced cost compared with conventional chemical vapor deposition techniques. The coating could be applied to the fiber by dip coating from a polymer solution, followed by pyrolysis to convert the coating to a ceramic. This could be done in an on-line process. In the polymeric state, the coating might serve as a sizing to protect the fiber during further handling and/or weaving, taking the place of a sizing that would require removal. This process could be 
applied to coating tows or, alternatively, might be used to coat cloth. The choice of a polymeric precursor, rheology and concentration of polymer solutions would be used to control coating thickness.

The present work focuses on surveying several potential precursor systems to establish initial parameters for coatings, using large diameter $(143 \mu \mathrm{m}) \mathrm{SCS}-0$ fibers. The use of large diameter fibers permits hand dipping of the fibers into the polymer solutions.

The polysilsesquioxane system has several specific advantages. First, it is easy to synthesize the polymer. It involves a simple lab bench procedure that can be easily done without any special equipment. The polymer is handled in air, making it readily adaptable to coating fibers on-line with few alterations to the process. The ability to change the ratios of monomers, which in turn changes the carbon content of the resulting polymer, is also beneficial. This allows tailoring interface properties to a specific fiber/matrix system.

Polyborosilazane derived Si-B-N ceramics offer the advantage of being oxidatively stable. This is necessary for the coatings to survive the anticipated use conditions. The ceramic remains amorphous to very high temperatures. Crystallization of the coating would result in a shrinkage, which would most likely lead to cracking, exposing the fiber to oxygen.

Polycarbosilane pyrolysed to carbon-rich silicon carbide. Crosslinking agents, such as 1,2-disilylethane, polybutadiene, trivinylmethylsilane and ammonia, can be easily incorporated into the polyvinylsilane. This allows for tailoring the polymer rheology and controlling curing.

\section{EXPERIMENTAL}

Polysilsesquioxanes were synthesized by copolymerizing a phenyl- and methyl- trimethoxysilane using a method described previously'. The ratio of the phenyl $(\mathrm{P})$ to methyl $(\mathrm{M})$ present in the polymer directly effected the carbon content of the final ceramic and also influenced the rheology. A greater percentage of phenyl in the polymer lowered the polymer viscosity and resulted in increased carbon in the ceramic. This study examines polymers with phenyl to methyl ratios of $5 / 95,10 / 90,30 / 70$ and $50 / 50$ to determine the effects of copolymer composition on the coating properties. After the polymers were synthesized, they were diluted in methanol to concentrations ranging between 515 percent, and SCS- 0 fibers were dipped into this solution. These coatings were then pyrolyzed in both argon and ammonia atmospheres at $1200^{\circ} \mathrm{C}$ for 30 minutes. Some of the fibers were then incorporated into a reaction bonded silicon 
nitride (RBSN) composite. The fibers were also characterized by scanning electron microscopy (SEM), Auger, and push out tests.

Two polyborosilazanes, synthesized by SRI International, were examined for use as coatings. The first polymer examined was synthesized by reacting borane with cyclomethylsilazane (CMS) in three different ratios of boron to silicon $(1: 8,1: 4$ and $1: 2)$. The polymerization of dichloromethylsilane and borontrichloride, at a boron to silicon ratio of $1: 4$, produced the second polymer examined. The fibers were coated from a 20 percent tetrahydrofuran solution. Coated fibers were characterized by SEM and Auger. Bulk powders were also prepared and characterized by thermogravimetric analysis (TGA), transmission electron microscopy (TEM) and $x$-ray diffraction (XRD).

Polyvinylsilane polymerization and pyrolysis is discussed in detail elsewhere ${ }^{2,3,4}$. Four different crosslinking agents have been examined for the vinylsilane polymer. They are 1,2-disilylethane (BSE), polybutadiene (PBD), trivinylmethylsilane (TVM) and ammonia $\left(\mathrm{NH}_{3}\right)$. The crosslinking agents help to tailor properties of the polymer and influence the observed viscosity of the material. The various compositions of the crosslinking agent would also be expected to somewhat alter the ceramic composition. Fibers were coated from a 10 percent cyclohexene solution.

\section{RESULTS AND DISCUSSION}

All of the polymers examined showed good fiber coverage by SEM. The coatings were smooth and virtually free of defects in all cases. Auger done on the fibers indicated that the coating thicknesses were usually in the range of $40-200$ nm.

Polysilsesquioxanes

The polysilsesquioxane system has been characterized in both the polymer and ceramic state in more detail previously ${ }^{1,5,6}$. The fiber coatings were examined to determine the effect of the carbon content and pyrolysis atmosphere on the final ceramic composition. Auger depth profiles showed that both carbon content and pyrolysis conditions had an influence on final polymer composition. After pyrolysis in argon the 50P/50M composition showed a large amount of carbon present in the coating ( Figure 1). In comparison, the $5 \mathrm{P} / 95 \mathrm{M}$ showed oxygen to be the predominant species. When the same compositions are examined after being pyrolyzed in ammonia, the $50 \mathrm{P} / 50 \mathrm{M}$ shows very little incorporation of nitrogen into the system, with the coating being primarily $\mathrm{SiO}_{2}$. The $5 \mathrm{P} / 95 \mathrm{M}$ on the other hand shows significant incorporation of nitrogen into the system, with significantly less oxygen present. Surface chemistry performed on the two 
coatings which had been pyrolyzed in ammonia also supported the finding that there was a proportionally larger amount of nitrogen present in the $5 \mathrm{P} / 95 \mathrm{M}$ derived coating. This difference in the amount of nitrogen incorporation can be explained by the way in which carbon is present in the two systems. The carbon present in the $5 \mathrm{P} / 95 \mathrm{M}$ has been shown to be predominately incorporated into the network. This is in contrast to the $50 \mathrm{P} / 50 \mathrm{M}$ polymer, in which a higher percentage of the carbon is in the form of free carbon. It was determined based on this information, that both silicon oxycarbides and silicon oxynitrides can be formed from the polysilsesquioxane polymer, depending on the pyrolysis environment.
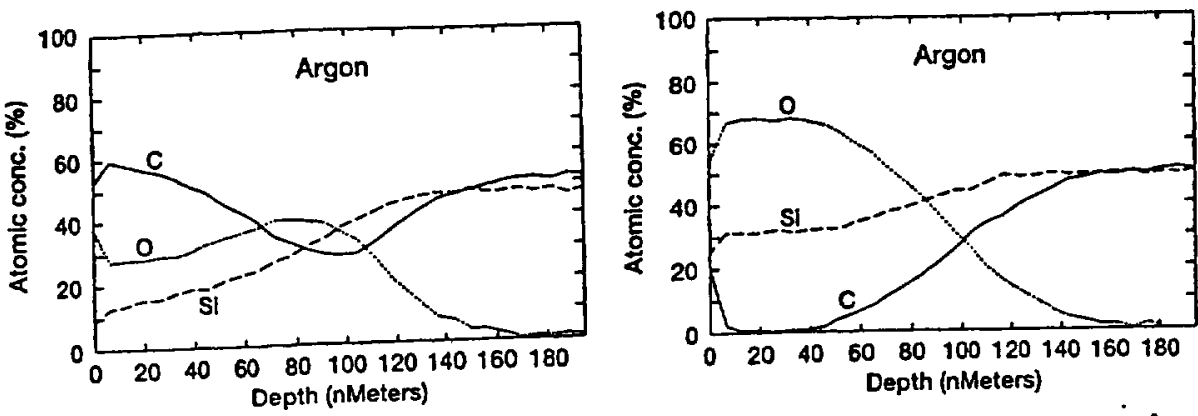

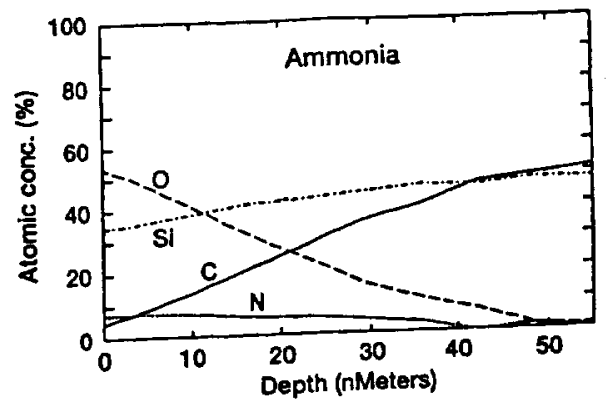

(A)

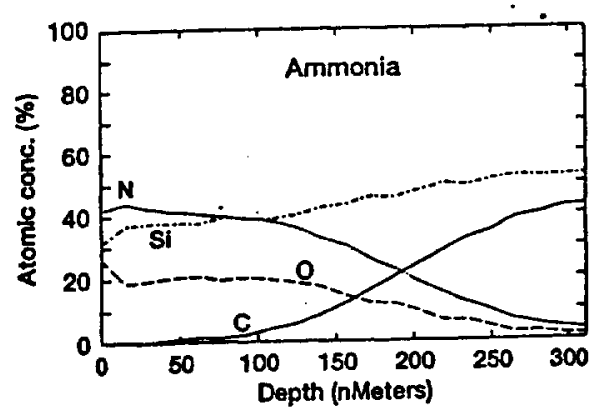

(B)

Figure 1. Auger depth profile of (A) 50P/50M and (B) $5 \mathrm{P} / 95 \mathrm{M}$ after pyrolysis in indicated atmosphere.

Fiber push out tests on fibers pyrolyzed in argon, then incorporated into a RBSN matrix, show that the coated fibers provide a weaker interface than the uncoated SCS-0 fibers, although it is still a stronger interface than desired. The coatings that were tested are felt to be too thin. It is expected that a weaker interface will result as the coating thicknesses are increased. Auger analysis of the coating that remained on the pushed out fiber showed evidence of nitrogen incorporation and no carbon. It was determined that the nitrogen must have been 
incorporated during the nitriding of the RBSN composite. For this reason, it is felt that the RBSN matrix is not an ideal model system to study these coatings. Future work will focus on studying the coatings in microcomposites fabricated by chemical vapor deposition (CVD) of a SiC matrix.

\section{Polyborosilazane}

The bulk ceramics formed by the two polyborosilazanes were examined to determine their potential effectiveness as a ceramic interface. Both polymers were found to yield $\mathrm{Si}-\mathrm{B}-\mathrm{N}$ ceramics. These ceramics were oxidatively stable after an initial pyrolysis in $\mathrm{NH}_{3}$ to $900^{\circ} \mathrm{C}$, followed by further heating in argon to temperatures of $1200^{\circ} \mathrm{C}$ or greater, with the weight loss at $1400^{\circ} \mathrm{C}$ in air being less than 1 percent (Figure 2). The ceramic that was only heated to $900^{\circ} \mathrm{C}$, was phase separated, so both the gold and black phases were tested for oxidative stability.

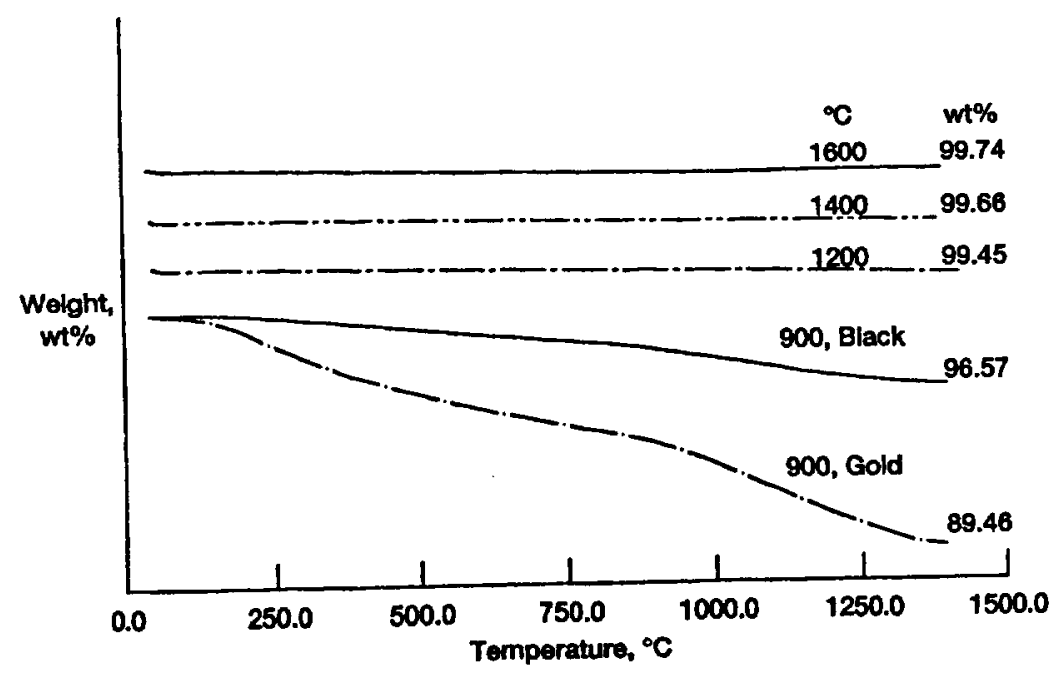

Figure 2. TGA profile of $\mathrm{MeHSiCl}_{2} / \mathrm{BCl}_{3}$ in air, after pyrolysis in argon to the indicated temperatures.

The effect of boron on the ceramic was also examined. Polycyclomethylsilazane (polymerization of CMS without boron) that had been heated to $1600^{\circ} \mathrm{C}$ was crystallized, primarily $\alpha-\mathrm{Si}_{3} \mathrm{~N}_{4}$ with some $\beta$. After boron incorporation, at a ratio of 1 boron for every 8 silicon atoms present, TEM results indicated that there was still areas that were crystalline $\beta-\mathrm{Si}_{3} \mathrm{~N}_{4}$ with some $\alpha$. There were also turbostratic areas in which the boron had inhibited crystallization. At a boron to silicon ratio of 1:4, the ceramic powder is shown by 
$\mathrm{X}$-ray diffraction to be amorphous for both of the polymer systems studied. The two polyborosilazanes examined result in a ceramic that offers the potential to provide a stable, amorphous fiber coating.

Polycarbosilane

The polyvinylsilane polymer and its resulting ceramic have been characterized in detail previously ${ }^{2,3,4}$. The polymer was shown to form a polycarbosilane backbone which resulted in an amorphous, carbon rich $\mathrm{Si}-\mathrm{C}$ upon pyrolysis in argon. The material, originally pyrolyzed in argon to $1400^{\circ} \mathrm{C}$ for 30 minutes, shows no weight change on reheating in air to the same temperature for 30 minutes. (Figure 3). This indicates the potential to provide a stable fiber coating. Work on this polymer system has also determined that smooth continuous coatings could be formed with the polymers when any of four crosslinking agents were added to the polyvinylsilane.

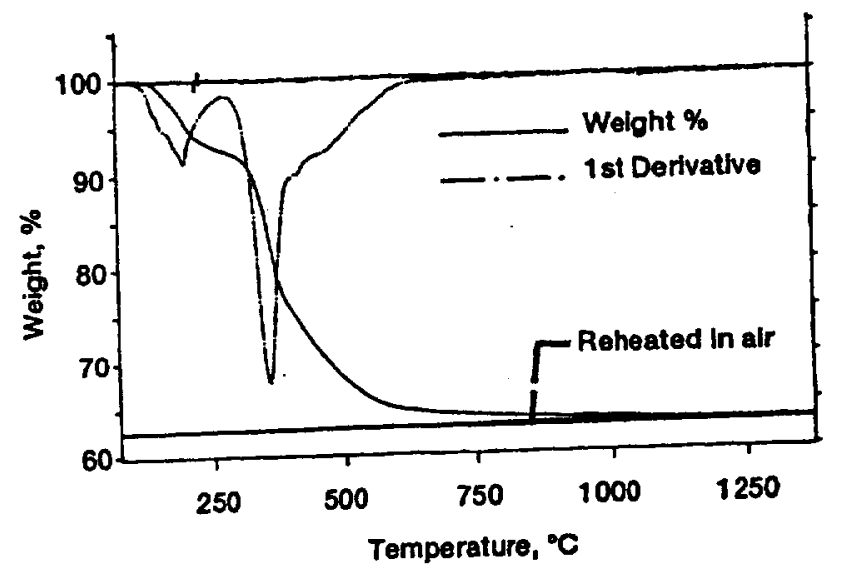

Figure 3. TGA of polyvinlysilane showing stability on reheating in air, after an initial pyrolysis in argon.

\section{CONCLUSIONS}

All of the polymer systems examined have resulted in smooth, thin fiber coatings on large diameter fibers. Pushout tests that were done on the polysilsesquioxane system show that the resulting ceramic does result in a weaker interface than uncoated SCS-0 fibers. These results should improve as both the composition and thickness of the coating is optimized. Both the polycarbosilane and polyborosilazane were found to be oxidatively stable to $1400^{\circ} \mathrm{C}$. This will provide for a stable coating under anticipated use conditions. The ability of the polyborosilazane to remain amorphous to high temperatures, means that it is 
unlikely that shrinkage will occur. Shrinkage of the coating would cause it to crack exposing the fiber to oxygen which would result in loss of fiber properties.

The final ceramic composition of each of the three polymers are different.

The polysilsesquioxane polymer results in a Si-C-O ceramic, the polyborosilazane forms a Si-B-N ceramic and the polysilsesquioxane pyrolyzes to a carbon-rich SiC. These differences in composition, and their related properties, could allow for better matching the requirements of a fiber/matrix system with an interface coating.

\section{REFERENCES}

1. P. J. Heimann, F. I. Hurwitz, A. L. River, "Synthesis of Polysilsesquioxanes for Use as Ceramic Precursors," Cream. Trans. 19 (Adv. Comps. Mater.), 27-33 (1991)

2. F. I. Hurwitz, T. A. Quack, X. Y. Bu, J. Mason, P. J. Heimann and K. Beyond, "Pyrolytic Conversion of Methyl- and Vinylsilane Polymers to Si-C Ceramics," submitted to $J$. Mater. Sci.

3. F. I. Hurwitz, T. A. Kacik, X. Y. Bu, J. Masnovi, P. J. Heimann and K. Beyene, "Conversion of Polymers of Methyl- and Vinylsilane to Si-C Ceramics," Mater. Res. Soc. Proc., to be published. Also NASA TM 106544 (1994).

4. J. Masnovi, X. Y. Bu, K. Beyene, P. J. Heimann, T. A. Kacik, A. H. Andrist and F. I. Hurwitz, "Syntheses, Structures and Properties of Polycarbosilanes Formed Directly by Polymerization of Alkenylsilanes," Mater. Res. Soc. Proc. 271, (Better Ceramics Through Chemistry V) 771-776 (1992) 5. F. I. Hurwitz, P. J. Heimann, J. Z. Gyekenyesi, J. Masnovi and X. Y. Bu, "Polymeric Routes to Silicon Carbide and Silicon Oxycarbide CMC," Ceram. Eng. Sci. Proc. 12, 1292-1303 (1991), also NASA TM 104372.

6. F. I. Hurwitz, P. J. Heimann, S. Farmer and D. M. Hembree, "Characterization of the Pyrolytic Conversion of Polysilsesquioxanes to Silicon Oxycarbides," J. Mater. Sci. 28, 6622-6630 (1993). 
